\title{
Spinal Modulation by Dexamethasone Sodium Phosphate, Cyclophosphamide and Miconazole Iontophoresis in asymptomatic SARS-COV2 patient with Extensive Myelitis
}

\section{Wassim Mushtaq*}

Neuro Rehablitation and Physiotherapy Clinic, Srinagar, India

*Corresponding Author: Wassim Mushtaq, Neuro Rehablitation and Physiotherapy Clinic, Srinagar, India.
Received: March 21, 2021

Published: April 12, 2021

(C) All rights are reserved by Wassim

Mushtaq.

\section{Abstract}

A very rare neurological complication of SARS-CoV-2 infection includes transverse myelitis. I assume a post-infectious etiology in terms of secondary immunogenic overreaction. Iontophoresis is the process of the permeation of ionic (charged) drugs into the body under the influence of electrical current. Besides increasing therapeutic efficiency by, by passing first pass metabolism there are less risks of systemic absorption and undesirable side effects. The study was conducted in a SARS-CoV-2 patient with transverse myelitis, by transdermal application of dexamethasone sodium phosphate, cyclophosphamide and miconazole by iontophoresis at corresponding vertebral levels to look for the neurological outcome who had been unresponsive to intravenous methylprednisolone. With Dexamethasone sodium phosphate and cyclophosphamide iontophoresis there was modulation of the activity of posterior grey column, fasiculus gracilis and corticospinal tracts, and with miconazole iontophoresis I was able to ameliorate the dyesthesias, fasiculations and muscle atrophy probably due to neuromodulation at substantia gelatinosa and lamina IX and remyelination effect. There were no systemic or localized side effects and no adverse effects occurred during the treatment period.

Keywords: Severe Acute Respiratory Syndrome Corona Virus-2 (SARS-CoV-2); Guillain-Barré Syndrome (GBS); Cerebro Spinal Fluid (CSF); Transverse Myelitis (TM); Reverse Transcriptase Polymerase Chain Reaction (RT-PCR); Interleukin (IL); Thoracic (T); Lumbar (L); Sacral (S); Intravenous (IV); Magnetic Resonance Imaging (MRI); Once Daily (OD); Neuromyelitis Optica (NMO); Anti Nuclear Antibody (ANA); AntiNeutrophil Cytoplasmic Antibodies (ANCA); Angiotensin Converting Enzyme (ACE); Veneral Disease Reference Laboratory (VDRL); Digital Subtraction Angiography (DSA); Herpes Simplex Virus (HSV); Epstein Barr Virus (EBV); Varicella Zoster Virus (VZV); CytoMegalo Virus (CMV); Human Immuno Deficiency Virus (HIV); Lipopolysaccharide (LPS); Regulated on Activation Normal T Cell Expressed (RANTES); Transforming Growth Factor Beta- $\beta 1$ (TGF- $\beta 1$ ); Nitric Oxide (NO); Macrophage Inflammatory Protein (MIP); Dexamethasone Sodium Phosphate (DEX-SP); Transforming Growth Factor B-Activated Kinase (TRAF); Jun N- Terminal Protein Kinase (JNK); InterLeukin Associated Kinase (IRAK); Pro-inflammatory T Helper (Th); Cytokine Interferon- $\gamma$ (IFN ${ }_{\gamma}$; Oligodendrocyte Progenitor Cells (OPCs); Cytochrome P450 Monooxygenase (CYP)

After the recognition of COVID-19 disease, caused by the SARSCoV-2, several reports refer to neurological symptoms in such patients [1,2], including Guillain-Barré Syndrome (GBS) [3-9]. A very rare neurological complication of SARS-CoV-2 infection includes transverse myelitis. Till date, a total of ten cases of transverse myelitis from acute SARS-CoV-2 infection have been reported (Sarma and Bilello, 2020; Zhao., et al. 2020; Chow., et al. 2020; Chakraborty., et al. 2020; Valiuddin., et al. 2020; AlKetbi., et al. 2020; Durrani., et al. 2020; Munz., et al. 2020; Zachariadis., et al. 2020; Abdelhady., et al. 2020) [53] In 30-60\% of idiopathic TM cases, there is an antecedent respiratory, gastrointestinal, or systemic illness [13]. I assume a post-infectious etiology in terms of secondary immunogen- 
ic overreaction. The association between COVID-19 and GBS has recently been described both as parainfectious [3,7] and as postinfective event $[4,5,9]$, similar to other infections and coronavirus $[11,12]$, suggesting a mechanism of molecular mimicry or part of systemic inflammatory cascade triggered by the virus.

Transverse myelitis has been attributed to infectious, parainfectious, systemic autoimmune diseases, paraneoplastic, and ischemic diseases (Joshi., et al. 2020; Kincaid and Lipton, 2020; Lycklama., et al. 2020; Borchers and Gershwin, 2020).

The diagnosis of trans-verse myelitis involves characteristic clinical presentation of bilateral signs and symptoms with a clearly defined sensory level, in addition to evidence on neuroimaging, CSF and serologic studies (Proposed diag-nostic criteria and nosology of acute transverse myelitis, 2002). This case report points towards COVID-19 as a possible cause of acute transverse myelitis [53].

A person in his late 40s with no underlying commorbidity had presented with a sudden onset headache, bilateral lower limb weakness and urinary incontinence. On RT- PCR the patient had been covid positive. His IL) Interleukin 6 levels were $(12 \mathrm{pg} / \mathrm{mL}$, normal $<5.9$ ), also associated with Covid-19 disease [10]. IL-6 is one of the primary cytokine involved in infection induced cytokinine storm. Patient had been managed as a case of myelopathy with a spinal shock and had received IV Methylprednisolone for 3 days and then the patient was transitioned to oral prednisone $60 \mathrm{mg}$ OD taper, for four months. His MRI dorsal spine showed diffused intramedullary/ edema involving the conus and mid lower dorsal cord. His CSF analysis showed moderate CSF pleocytosis with neutrophilic predominance. CSF protein content was mildly raised, with normal sugar levels. LDH levels in the CSF were markedly raised. CBC was within normal limits. Anti NMO antibodies and oligoclonal bands were negative in both CSF and serum. Anticardiolipin and phospholipid antibodies were negative too. Vasculitic profile including ANA, ANCA, ACE levels were within normal limits. VDRL and viral markers were non reactive. His DSA and spinal angiogram was normal. Microbiologic testing on CSF and serum was negative (HSV1-2, EBV, VZV, CMV, HIV, Mycoplasma Pneumoniae, Borrelia).

Before starting with transdermal dorsal vertebral iontophoresis, 5 months after the sequale the patients neurological examination revealed diminished sensation, in the dermatome T8- T12, with the loss of all superficial, deep and combined cortical sensations except deep touch sensation till T12 dermatome, with a Medi- cal Research Council (MRC) scale grading of muscle power as 3/5 in the abdominal muscles, 2/5 in the quadrates lamborum and 0/5 in the lower limbs. The patient had a flaccidity in his lower limbs. The patient was complaining of dysthesias and persistent fasiculations in his lower limbs. There was no bladder or bowel control. The abdominal reflex was present, $\mathrm{B} / \mathrm{l}$ knee reflexes were absent and ankle reflex was present. The plantars were extensors in nature.

Dexamethasone sodium phosphate $(4 \mathrm{mg})$ and cyclophosphamide $(250 \mathrm{mg}$ ) transdermal iontophoresis on corresponding vertebral segments (T9-T12) and T12-L2 was initially initiated for first three weeks followed by iontophoresis by miconazole nitrate gel $2 \%$ and polyethylene glycol for next three weeks to look for the outcome. On day 3 of the iontophoresis the plantars were down going, On day 4 pelvic control started developing. On Day 5 knee extensors and ankle dorsiflexors were grade 1, On day 10 the patient gained Knee and ankle joint proprioception and the patient responded to deep pressure sense at L5-S1, S1-S2, S3- S4 dermatome. On Day 15 pelvic control of Grade 3 was achieved. On Day 22 we started with the transdermal iontophoresis of Micanazole nitrate gel $2 \%$ to look for the outcome. On day $28^{\text {th }}$ Pelvic Control of Grade 4 was achieved and by day $35^{\text {th }}$, the patient had a full relief from dyesthesias and fibrillations. His long toe flexors and dorsiflexors, knee extensors, hip extensors were grade 1 and there was a slight increase in muscle tone.

\section{Discussion}

Dexamethasone helps to reduce inflammation and calms down an overactive immune system. The exact mechanism of action is not clear but some of the possible mechanisms include antiedema effect, stabilization of blood brain barrier, reduction of proinflammotory cytokines and apoptosis of T cells [54]. Dexamethasone sodium phosphate (Dex-P) delivered via iontophoresis is commonly used in physiotherapy to treat tendinopathies; bursitis, shin splints, rheumatoid arthritis and delayed muscle soreness [1436]. Dexamethasone acts as a stimulator of glucocorticoid receptors signaling pathway, which reduces the production of cytokines and decreases neutrophil tissue invasion and damage. In addition, dexamethasone inhibits the phospholipase A2 enzyme that plays a role in the biosynthesis of leukotrienes, prostaglandins, thromboxane A2, and prostacyclin [37]. It has been found that abnormal neuroinflammation ignited by overproduction of chemokines and cytokines via microglial cells can induce the occurrence and de- 
Spinal Modulation by Dexamethasone Sodium Phosphate, Cyclophosphamide and Miconazole Iontophoresis in asymptomatic SARS-COV2 patient with Extensive Myelitis

velopment of neurodegenerative disorders. It has been found that dexamethasone sodium phosphate inhibited the neuroinflammatory response and migration in LPS Lipopolysaccharide-activated BV-2 microglia by inhibiting the secretion of RANTES, as regulated on activation, normal $\mathrm{T}$ cell expressed and secreted transforming growth factor beta- $\beta 1$ TGF- $\beta 1$, and NO nitric oxide and increasing the production of MIP- $1 \alpha$ macrophage inflammatory protein- $1 \alpha$ and interleukin 10 IL-10, besides inhibition of TRAF6/TAK-1/JNK tumor necrosis factor receptor-associated factor 6/ Transforming growth factor B- activated kinase 1/ Jun N- terminal protein Kinase signaling pathways mediated by IRAK-1 and IRAK-4 (InterLeukin Associated Kinase 1 and 4) [38].

In patients with severe acute immune-mediated longitudinally extensive transverse myelitis who fail to respond to corticosteroids and plasma exchange, cyclophosphamide induction should be considered [39]. This agent and regimen provides a robust immunosuppressive response and can be induced rapidly. Cyclophosphamide has been shown to decrease the secretion of the pro-inflammatory T helper (Th) 1 cytokine interferon- $\gamma$ (IFN $\gamma$ ) and interleukin (IL)12 and to increase the secretion of the anti-inflammatory Th2 cytokines IL-4 and IL-10 in cerebrospinal fluid (CSF) and peripheral blood. Methotrexate which is also a immunosuppressant and chemotherapy agent has been administered through iontophoresis by using hydrogel patches [40]. It was found that the transport was influenced by physicochemical properties of the system (crosslinking density of the hydrogel and copolymerisation), duration of electrical currents and the condition of the skin. Besides this the iontophoretic application of cyclophosphamide has been used to treat breast cancer and mastitis [41]. Further in a study Cyclophosphamide administration in association with osmotic blood-brain barrier opening did not cause significant neural toxicity [42].

Based on the defined role of dexamethasone and cyclophosphamide as chemical neuromodulators, neuromodulation by way of iontophoresis in asymptomatic SARS-COV 2 with extensive myelitis was considered to look for outcome. The other considerations which were kept in view were rate of iontophoretic delivery and the chemical property of Cyclophosphamide as it is inert until activated by enzymes in the liver. Cyclophosphamide is biotransformed principally in the liver to active alkylating metabolites by a mixed function microsomal oxidase system. In the present study, the levels of the plasma concentration of the drug reached, which might have passed into systemic circulation through dermal capillary network to liver to undergo activation remains unclear. Complete blood count including Liver Function, Kidney Function Tests were carried out on weekly basis to rule out any toxicity due to cyclophosphamide iontophoresis, and the parameters were normal.

Studies designed to evaluate the depth of penetration of the drugs (DEX, prednisolone, salicylate, and lidocaine) into local tissue following iontophoresis have demonstrated that a depot is formed in the area of the epidermis [43-46]. Deeper penetration of the drug apparently occurs not from iontophoretic current, but from passive diffusion. Passive diffusion is a slower, mass transfer process compared with iontophoresis. Thus, for equivalent iontophoretic dosages, it is time, not current magnitude, that dictates the ultimate local depth of penetration. In living tissue, however, other factors such as local blood flow will determine the ultimate depth of local penetration. A study conducted [47] to determine the time course of dexamethasone sodium phosphate (Dex-P) during iontophoresis to underlying tissues using microdialysis there was no difference in Dex-total between current intensities $(\mathrm{P}=$ .99), but a greater amount of Dex-total was recovered superficially at $1 \mathrm{~mm}$ compared to the 4-mm depth (P<.0001). 17 I In our study we used $40 \mathrm{~mA}$ plain faradic current delivered for $10 \mathrm{~min}$ utes to reach a therapeutic effect. The dexamethasone was delivered through cathode and cyclophosphamide through anode. Metallic electrodes secured with lint pads which were instilled with drugs were used for iontophoresis. Cyclophosphamide $250 \mathrm{mg}$ was diluted with $20 \mathrm{ml}$ saline. Through our study we were able to modulate the activity of posterior grey column, fasciculus gracilis and corticospinal tracts which was apparent as the patient gained joint proprioception of the knee and ankle and responded to the deep pressure sense at L5-S1, S1-S2,S3-S4 dermatome, down going plantars and attaining a pelvic control of Grade 3 in quadrapod position. It has been found that the antifungal miconazole and the steroid clobetasol stimulates mouse and human oligodendrocyte progenitor cells (OPCs) into generating myelin-producing cells in culture. $[48,49]$. They found that miconazole and eight other related drugs all blocked an enzyme called CYP51. Blocking CYP51 encouraged stem cells to form new oligodendrocytes. These are the cells that create the myelin coatings around nerve cells.

CYP51 is part of the molecular pathway that produces cholesterol. The researchers discovered that blocking two other enzymes in that pathway also promoted oligodendrocyte production. The utilization of chemical penetration enhancers in conjunction with iontophoresis is regarded as the most effective method to enhance the passage of molecules across the skin barrier 50. In our study 
we used polyvinyl alcohol [51,52] to enhance the permeability of Miconazole. Transdermal iontophoretic application of miconazole nitrate for three weeks was able to ameliorate dyesthesias, fasiculations and muscle atrophy probably due to neuromodulation at substantia gelatinosa and lamina IX and remyelination effect.

\section{Conclusion}

Dexamethasone Sodium Phosphate, Cyclophosphamide and Miconazole Iontophoresis on subsequent transdermal spinal levels can be considered in patients with myelitis, for modulation, during acute stages.

\section{Bibliography}

1. Wang HY., et al. "Potential neurological symptoms of COVID-19". Therapeutic Advances in Neurological Disorders (2020).

2. Huang C., et al. "Clinical features of patients infected with 2019 novel coronavirus in Wuhan, China". Lancet 395.10223 (2020): 497-506.

3. Zhao H., et al. "Guillain-Barré syndrome associated with SARSCoV-2 infection: causality or coincidence?" Lancet Neurology 19.5 (2020): 383-384.

4. Toscano G., et al. "Guillain-Barrè syndrome associated with Sars-CoV-2". The New England Journal of Medicine (2020).

5. Padroni M., et al. "Guillain-Barré syndrome following COVID-19: new infection, old complication?" Journal of Neurology 24 (2020): 1-3.

6. Virani A., et al. "Guillain-Barré syndrome associated with SARS-CoV-2 infection”. IDCases 18.20 (2020): e00771.

7. Ottaviani D., et al. "Early Guillain-Barré syndrome in Coronavirus Disease 2019 (COVID-19): a case report from an Italian COVID-hospital". Neurology Science 41.6 (2020): 1351-1354.

8. Juliao Caamaño DS and Alonso BR. "Facial diplegia, a possible atypical variant of Guillain-Barré Syndrome as a rare neurological complication of SARS-CoV-2". Journal of Clinical Neuroscience (2020).

9. Sedaghat $\mathrm{Z}$ and Karimi N. "Guillan-Barré syndrome associated with COVID-19 infection: A case report". Journal of Clinical Neuroscience 76 (2020): 233-235.

10. Guan WJ., et al. "China medical treatment expert group for Covid-19. Clinical characteristics of coronavirus disease 2019 in China". The New England Journal of Medicine 382.18 (2020): 1708-1720.
11. Uncini A., et al. "Zika virus infection and GuillainBarré syndrome: a review focused on clinical and electrophysiological subtype". Journal of Neurology, Neurosurgery, and Psychiatry 88 (2017): 266-271.

12. Kim JE., et al. "Neurological complications during treatment of middle east respiratory syndrome". Journal of Clinical Neurology 13 (2017): 227-233.

13. Krishnan C., et al. "Transverse Myelitis: pathogenesis, diagnosis and treatment". Frontiers in Bioscience 9 (2004): 14831499.

14. Amirjani N., et al. "Corticosteroid iontophoresis to treat carpal tunnel syndrome: a double-blind randomized controlled trial”. Muscle Nerve 39 (2009): 627-633.

15. Anderson CR., et al. "Effects of iontophoresis current magnitude and duration on dexamethasone deposition and localized drug retention". Physical Therapy 83 (2003): 161-170.

16. Berliner MN. "Skin microcirculation during tapwater iontophoresis in humans: cathode stimulates more than anode". Microvascular Research 54 (1997): 74-80.

17. Pellecchia GL., et al. "Treatment of infrapatellar tendinitis: a combination of modalities and transverse friction massage versus iontophoresis". Journal of Sport Rehabilitation 3 (1994): 135-145.

18. Phipps JB., et al. "Iontophoretic delivery of model inorganic and drug ions". Journal of Pharmaceutical Sciences 78 (1989): 365-369.

19. Riviere JE., et al. "Determination of lidocaine concentrations in skin after transdermal iontophoresis: effects of vasoactive drugs". Pharmaceutical Research 9 (1992): 211-214.

20. Runeson L and Haker E. "Iontophoresis with cortisone in the treatment of lateral epicondylalgia (tennis elbow) - a doubleblind study". Scandinavian Journal of Medicine and Science in Sports 12 (2002): 136-142.

21. Smutok MA., et al. "Failure to detect dexamethasone phosphate in the local venous blood postcathodic lontophoresis in humans". Journal of Orthopaedic and Sports Physical Therapy 32 (2002): 461-468.

22. Stahl M., et al. "Human microdialysis". Current Pharmaceutical Biotechnology 3 (2002): 165-178. 
23. Stefanou A., et al. "A randomized study comparing corticosteroid injection to corticosteroid iontophoresis for lateral epicondylitis". Journal of Hand Surgery (American Volume) 37 (2012): 104-109.

24. Blackford J., et al. "Iontophoresis of dexamethasone-phosphate into the equine tibiotarsal joint". Journal of Veterinary Pharmacology and Therapeutics 23 (2000): 229-236.

25. Coglianese M., et al. "Microdialysis and delivery of iontophoresis-driven lidocaine into the human gastrocnemius muscle". Journal of Athletic Training 46 (2011): 270-276.

26. Draper DO., et al. "Absorption of iontophoresis-driven 2\% lidocaine with epinephrine in the tissues at $5 \mathrm{~mm}$ below the surface of the skin". Journal of Athletic Training 46 (2011): 277-281.

27. Grossmann M., et al. "The effect of iontophoresis on the cutaneous vasculature: evidence for current-induced hyperemia". Microvascular Research 50 (1995): 444-452.

28. Gurney $\mathrm{AB}$ and Wascher DC. "Absorption of dexamethasone sodium phosphate in human connective tissue using iontophoresis". American Journal of Sports Medicine 36 (2008): 753759.

29. Gurney B., et al. "The effect of skin thickness and time in the absorption of dexamethasone in human tendons using iontophoresis". Journal of Orthopaedic and Sports Physical Therapy 38 (2008): 238-245.

30. Guy RH., et al. "Iontophoresis: electrorepulsion and electroosmosis". Journal of Control Release 64 (2000): 129-132.

31. Heiss JD., et al. "Mechanism of dexamethasone suppression of brain tumor-associated vascular permeability in rats. Involvement of the glucocorticoid receptor and vascular permeability factor". Journal of Clinical Investigation 98 (1996): 1400-1408.

32. Hong SU., et al. "Pharmacological evaluation of five novel dexamethasone heneicosoic (21) acid derivatives". Drugs Under Experimental and Clinical Research 15 (1989): 511-520.

33. James MP., et al. "Percutaneous iontophoresis of prednisolone - a pharmacokinetic study". Clinical and Experimental Dermatology 11 (1986): 54-61.

34. Neeter C., et al. "Iontophoresis with or without dexamethazone in the treatment of acute Achilles tendon pain". Scandinavian Journal of Medicine and Science in Sports 13 (2003): 376-382.
35. Nirschl RP., et al. "Iontophoretic administration of dexamethasone sodium phosphate for acute epicondylitis. A randomized, double-blinded, placebo-controlled study". American Journal of Sports Medicine 31 (2003): 189-195.

36. Osborne HR and Allison GT. "Treatment of plantar fasciitis by LowDye taping and iontophoresis: short term results of a double blinded, randomised, placebo controlled clinical trial of dexamethasone and acetic acid". British Journal of Sports Medicine 40 (2006): 545-549; discussion 549.

37. Dr. Sulaiman Al Habib. "Dexamethasone as a Treatment of COVID-19”. Medical Journal 3.1 (2021): 7-9.

38. Bin Hui., et al. "Dexamethasone sodium phosphate attenuates lipopolysaccharide-induced neuroinflammation in microglia BV2 cells". Naunyn-Schmiedeberg's Archives of Pharmacology 393 (2020): 1761-1768.

39. Laura J Baxter., et al. "Refractory Longitudinally Extensive Transverse Myelitis Responsive to Cyclophosphamide". Canadian Journal of Neurological Sciences 44.6 (2017): 736-739.

40. Prasad R., et al. "Transdermal iontophoretic delivery of methotrexate: physicochemical considerations". Trends in Biomaterials and Artificial Organs 18.2 (2005): 18-25.

41. Nagoya-shi Aichi JP. "Iontophoretic preparation for treatment of breast cancer and or mastitis". United States Patent Application 20090281063Kowa Co., Ltd. Tokyo JP. Tokyo university of science educational foundation administrative organization.

42. Pharmacology and neurotoxicity of cis-diamminedichloroplatinum, bleomycin, 5-fluorouracil, and cyclophosphamide administration following osmotic blood-brain barrier modification.

43. Glass JM., et al. "The quantity and distribution of radiolabeled dexamethasone delivered to tissue by iontophoresis". International Journal of Dermatology 19.9 (1980): 519-525.

44. James MP., et al. "Percutaneous iontophoresis of prednisolone: a pharmacokinetic study". Clinical and Experimental Dermatology (1986): 54-61.

45. Riviere JE., et al. "Determination of lidocaine concentrations in skin after transdermal iontophoresis: effects of vasoactive drugs". Pharmaceutical Research 9 (1992): 211-214.

46. Singh P and Roberts MS. "Iontophoretic transdermal delivery of salicylic acid and lidocaine to local subcutaneous structures". Journal of Pharmaceutical Sciences 82 (1993): 127-131. 
47. Justin H Rigby., et al. "The time course of dexamethasone delivery using iontophoresis through human skin, measured via microdialysis". Journal of Orthopaedic and Sports Physical Therapy 45.3 (2015): 190-197.

48. Nature, along with a companion methods paper in Nature Methods (2018).

49. Ryan Hartwell., et al. "Polyvinyl alcohol-graft-polyethylene glycol hydrogels improve utility and biofunctionality of injectable collagen biomaterials". Biomed Materials 11.3 (2016): 035013.

50. Nair A., et al. "Effect of permeation enhancers on the iontophoretic transport of metoprolol tartrate and the drug retention in skin". Drug Delivery 8 (2011): 19-25.

51. Ryan Hartwell., et al. "Polyvinyl alcohol-graft-polyethylene glycol hydrogels improve utility and biofunctionality of injectable collagen biomaterials". Biomed Materials 11.3 (2016): 035013.

52. Andrea Porzionato., et al. "Development of Oxidized Polyvinyl Alcohol-Based Nerve Conduits Coupled with the Ciliary Neurotrophic Factor". Materials 12.12 (2019): 1996.

53. Maria CamilaMoren., et al. "Acute transverse myelitis with Dysautonomia following SARS-CoV-2 infection: A case report and review of literature". Journal of Neuroimmunology 353 (2021): 577523.

54. R Gold. "Mechanism of action of glucocorticosteroid hormones: possible implications for therapy of neuroimmunological disorders". Journal of Neuroimmunology 17 (2001): 1-8.

\section{Assets from publication with us}

- Prompt Acknowledgement after receiving the article

- Thorough Double blinded peer review

- Rapid Publication

- Issue of Publication Certificate

- High visibility of your Published work

Website: www.actascientific.com/

Submit Article: www.actascientific.com/submission.php

Email us: editor@actascientific.com

Contact us: +919182824667 